\title{
The two-block covariance matrix and the CAPM
}

\section{David Disatnik* and Simon Benninga}

Faculty of Management,

Tel Aviv University,

Tel Aviv, 69978, Israel

E-mail: daviddis@post.tau.ac.il

E-mail: benninga@post.tau.ac.il

*Corresponding author

\begin{abstract}
The classical assumptions of the capital asset pricing model do not ensure obtaining a tangency (market) portfolio in which all the risky assets appear with positive proportions. This paper gives an additional set of assumptions that ensure obtaining such a portfolio. Our new set of assumptions mainly deals with the structure of the covariance matrix of the risky assets returns. The structure we suggest for the covariance matrix is of a two-block type. We derive analytically sufficient conditions for a matrix of this type to produce a long-only tangency portfolio (as well as a long-only global minimum variance portfolio).
\end{abstract}

Keywords: portfolio optimisation; block covariance matrix; tangency portfolio; market portfolio; capital asset pricing model; CAPM.

Reference to this paper should be made as follows: Disatnik, D. and Benninga, S. (2012) 'The two-block covariance matrix and the CAPM', Int. J. Portfolio Analysis and Management, Vol. 1, No. 1, pp.32-42.

Biographical notes: David Disatnik is an Assistant Professor of Accounting at Tel Aviv University. He received his BA in Accounting and Economics from Tel Aviv University; MBA in Finance from the Hebrew University in Jerusalem; and $\mathrm{PhD}$ in Accounting and Finance from Tel Aviv University. Prior to joining the Faculty of Management at Tel Aviv University, he taught in the Ross School of Business at the University of Michigan.

Simon Benninga is a Professor of Finance at Tel Aviv University. From 1993-2003 he edited the Review of Finance, the Journal of the European Finance Association, which he founded. He is the author of over 40 academic papers and of four books: Numerical Techniques in Finance, Corporate Finance: A Valuation Approach, Financial Modeling, and Principles of Finance with Excel.

\section{Introduction}

The Markowitz $(1952,1959)$ model is one of the great intellectual achievements of 20th century finance. Markowitz formalised the concept of diversification and his model led directly to the capital asset pricing model (CAPM) of Sharpe (1964), Lintner(1965), and Mossin (1966). Nobel prizes in economics (i.e., Markowitz, Sharpe, and Tobin) are due wholly or in part to this massive, path-breaking paradigm. 
While a magnificent intellectual achievement, the Markowitz/CAPM is one of the most non-implemented models of the 20th century revolution in finance. Though the model has been used for attractive tests of capital market efficiency and pricing, it has failed to fulfil its original mission - to be a viable guide in choosing an optimal portfolio. Compared to that other blockbuster intellectual achievement of finance, the option pricing model of Black, Scholes, and Merton, the Markowitz model is rarely used, and -when used in its original form - almost always fails to lead to plausible portfolio choices.

According to the seminal work of Markowitz $(1952,1959)$ an investor who cares only about the mean and variance of portfolio returns should hold a portfolio on the efficient frontier. In practice, applying the Markowitz mean-variance theory involves estimating the means and covariances of asset returns, and often results in portfolios with large short sale positions. This is true both when the means and covariances are estimated by the traditional sample mean vector and the sample covariance matrix respectively, as well as by more advanced estimation techniques. ${ }^{1}$ Obtaining portfolios with short sale positions can be considered a major drawback, since short selling is often restricted by regulators, investment policies of mutual funds sometimes prohibit taking short positions, and many individual investors find short selling onerous or impossible. ${ }^{2}$ In addition, whereas asset positions in a long-only portfolio, and hence turnover and transactions costs, are strictly bounded, this is not true for portfolios with short positions.

Of the portfolios on the efficient frontier, two are of a particular interest. The first is the tangency portfolio, since, given the assumptions of the CAPM and in the presence of a risk-free asset, the CAPM identifies the tangency portfolio with the market portfolio. Thus, from the CAPM perspective, obtaining a tangency portfolio that includes short sale positions is problematic, since it precludes its identification with the market portfolio.

The second efficient portfolio of interest is the global minimum variance portfolio (henceforth - GMVP), which is, in the mean-variance framework, the portfolio on the efficient frontier with the smallest return variance. The interest in the GMVP stems from the fact that the derivation of the GMVP requires estimating only the covariance matrix of asset returns, whereas for other efficient portfolios we have to estimate the means of asset returns as well, and that significantly adds to the estimation error. However, also in the GMVP case, its derivation in practice using the Markowitz framework results in obtaining highly illogical portfolios with significant short sale positions. ${ }^{3}$

Almost from inception it was realised that the failures of the Markowitz/CAPM model as a prescriptive instrument for portfolio choice have their roots in the incompatibility of historical asset return data with the inputs required for the model (the vector of expected returns and the covariance matrix of asset returns). As anyone who has ever tried a naïve implementation of Markowitz on almost any historical dataset soon realises, data is a poor predictor of expected returns and covariances (correlations), and this is what leads to the above mentioned implausible portfolios with the significant short positions. ${ }^{4}$

\subsection{A numerical example}

We illustrate the difficulties with the Markovitz model with an example. Consider annual return data for 1994-2004 for nine arbitrary US stocks (General Electric, Microsoft, Johnson \& Johnson, Kellogg, Boeing, 3M, IBM, Intel, and Merck): 
Figure 1 Illustration of the difficulties with the Markowitz model

\begin{tabular}{|c|c|c|c|c|c|c|c|c|c|c|}
\hline & $\mathrm{A}$ & $\mathrm{B}$ & $\mathrm{C}$ & $\mathrm{D}$ & $E$ & $\mathrm{~F}$ & $\bar{G}$ & $\mathrm{H}$ & $T$ & $J$ \\
\hline 1 & \multicolumn{10}{|c|}{ NINE STOCKS, ANNUAL DATA, 1994-2004 } \\
\hline 2 & $\begin{array}{l}\text { Percentage } \\
\text { of portfolio }\end{array}$ & $24.28 \%$ & $22.07 \%$ & $11.03 \%$ & $1.11 \%$ & $2.96 \%$ & $5.24 \%$ & $9.93 \%$ & $15.79 \%$ & $7.58 \%$ \\
\hline 3 & $\begin{array}{l}\text { Average } \\
\text { return }\end{array}$ & $23.66 \%$ & $21.38 \%$ & $18.43 \%$ & $5.51 \%$ & $27.63 \%$ & $21.14 \%$ & $17.63 \%$ & $23.05 \%$ & $13.81 \%$ \\
\hline 4 & $\begin{array}{l}\text { Standard } \\
\text { deviation }\end{array}$ & $32.17 \%$ & $40.71 \%$ & $18.97 \%$ & $23.86 \%$ & $29.93 \%$ & $25.84 \%$ & $13.56 \%$ & $52.19 \%$ & $25.18 \%$ \\
\hline 5 & & & & & & & & & & \\
\hline 6 & \multicolumn{10}{|c|}{ Variance-covariance matrix } \\
\hline 7 & & GE & MSFT & JNJ & $\mathbf{K}$ & BA & MMM & IBM & INTC & MRK \\
\hline 8 & GE & 0.0941 & 0.0690 & 0.0201 & -0.0039 & 0.0780 & 0.0612 & 0.0112 & 0.1144 & 0.0467 \\
\hline 9 & MSFT & 0.0690 & 0.1507 & 0.0375 & -0.0047 & 0.0345 & 0.0827 & -0.0020 & 0.1460 & 0.0548 \\
\hline 10 & JNJ & 0.0201 & 0.0375 & 0.0327 & 0.0165 & 0.0092 & 0.0270 & -0.0036 & 0.0261 & 0.0291 \\
\hline 11 & $\mathrm{~K}$ & -0.0039 & -0.0047 & 0.0165 & 0.0518 & -0.0069 & -0.0074 & -0.0042 & -0.0241 & 0.0103 \\
\hline 12 & BA & 0.0780 & 0.0345 & 0.0092 & -0.0069 & 0.0814 & 0.0399 & 0.0226 & 0.0945 & 0.0388 \\
\hline 13 & MMM & 0.0612 & 0.0827 & 0.0270 & -0.0074 & 0.0399 & 0.0607 & 0.0001 & 0.0965 & 0.0408 \\
\hline 14 & IBM & 0.0112 & -0.0020 & -0.0036 & -0.0042 & 0.0226 & 0.0001 & 0.0167 & 0.0304 & 0.0049 \\
\hline 15 & INTC & 0.1144 & 0.1460 & 0.0261 & -0.0241 & 0.0945 & 0.0965 & 0.0304 & 0.2476 & 0.0466 \\
\hline 16 & MRK & 0.0467 & 0.0548 & 0.0291 & 0.0103 & 0.0388 & 0.0408 & 0.0049 & 0.0466 & 0.0577 \\
\hline 17 & & & & & & & & & & \\
\hline 18 & \begin{tabular}{|l} 
Risk-free \\
rate
\end{tabular} & $3 \%$ & & & & & & & & \\
\hline 19 & & & & & & & & & & \\
\hline 20 & & $\begin{array}{c}\text { Tangency } \\
\text { portfolio }\end{array}$ & GMVP & & \multirow{8}{*}{\multicolumn{6}{|c|}{$\begin{array}{l}\text { Explanation: The return data in row } 3 \text { is annual for 1994- } \\
\text { 2004. Row } 2 \text { shows the value weights of the portfolio of } \\
\text { these } 9 \text { stocks using market values at the end of } 2004 \text {. } \\
\text { The risk-free rate of } 3.0 \% \text { is the approximate annual } \\
\text { return on U.S. Treasury securities at the end of } 2004 \text {. }\end{array}$}} \\
\hline 21 & GE & $8.63 \%$ & $12.57 \%$ & & & & & & & \\
\hline 22 & MSFT & $20.97 \%$ & $18.08 \%$ & & & & & & & \\
\hline 23 & JNJ & $2.33 \%$ & $-0.04 \%$ & & & & & & & \\
\hline 24 & $\mathrm{~K}$ & $7.18 \%$ & $8.03 \%$ & & & & & & & \\
\hline 25 & BA & $-0.69 \%$ & $-7.94 \%$ & & & & & & & \\
\hline 26 & MMM & $57.35 \%$ & $57.10 \%$ & & & & & & & \\
\hline 27 & IBM & $88.48 \%$ & $90.30 \%$ & & & & & & & \\
\hline 28 & INTC & $-40.30 \%$ & $-38.27 \%$ & & & & & & & \\
\hline 29 & MRK & $-43.97 \%$ & $-39.82 \%$ & & & & & & & \\
\hline
\end{tabular}

We can see that the Markowitz optimisation produces a tangency portfolio whose weights (column B, rows 21-29) differ significantly from the value weights of the portfolio of the nine stocks using market values at the end of 2004 (row 2). Moreover, there are three negative positions in the computed tangency portfolio, two of which are very large (Intel and Merck). ${ }^{5}$ As to the GMVP, it has four negative portfolio positions. In most practical optimisation situations, this of course would be impossible to implement.

The correlation matrix of the returns reveals another problem: The historical data has three correlations above 0.8 . While this is undoubtedly a property of the data, it is difficult to believe that this is also a forward-looking correlation.

Figure 2 Correlation data

\begin{tabular}{|c|c|c|c|c|c|c|c|c|c|c|}
\hline & $\mathrm{A}$ & $\mathrm{B}$ & $\mathrm{C}$ & $\mathrm{D}$ & $E$ & $\mathrm{~F}$ & $\mathrm{G}$ & $\mathrm{H}$ & I & $\mathrm{J}$ \\
\hline 32 & \multicolumn{10}{|c|}{ Correlation Matrix } \\
\hline 33 & & GE & MSFT & JNJ & K & BA & MMM & IBM & INTC & MRK \\
\hline 34 & GE & 1.0000 & 0.5791 & 0.3632 & -0.0560 & 0.8905 & 0.8100 & 0.2819 & 0.7492 & 0.6334 \\
\hline 35 & MSFT & & 1.0000 & 0.5340 & -0.0532 & 0.3113 & 0.8647 & -0.0406 & 0.7556 & 0.5880 \\
\hline 36 & JNJ & & & 1.0000 & 0.4002 & 0.1780 & 0.6052 & -0.1529 & 0.2900 & 0.6699 \\
\hline 37 & $\mathbf{K}$ & & & & 1.0000 & \begin{tabular}{|c|}
-0.1067 \\
\end{tabular} & -0.1319 & -0.1427 & -0.2131 & 0.1880 \\
\hline 38 & BA & & & & & 1.0000 & 0.5667 & 0.6116 & 0.6657 & 0.5669 \\
\hline 39 & MMM & & & & & & 1.0000 & 0.0040 & 0.7866 & 0.6903 \\
\hline 40 & IBM & & & & & & & 1.0000 & 0.4721 & 0.1590 \\
\hline 41 & INTC & & & & & & & & 1.0000 & 0.3899 \\
\hline 42 & MRK & & & & & & & & & 1.0000 \\
\hline
\end{tabular}




\subsection{Is there a solution?}

The problematics of the Markowitz model were realised almost from the first and led to a variety of workarounds that initially focused mostly on the covariance matrix. The single-index model of Sharpe (1963) and Elton-Gruber's (1973) constant correlation model can be seen as two of these workarounds. Both of these models, which stem from the early days of the CAPM, ignore covariances computed from the data and substitute instead alternative assumptions about how covariances can be computed. In the case of the single-index model, the assumption is that the co-movement between risky assets is due to a single common influence or index. In the case of the constant correlation model, the assumption is that every pair of risky assets has the same correlation coefficient. ${ }^{6}$

An early alternative to the problem of computing the expected returns is Sharpe's (1974) "reverse engineering” of expected returns. ${ }^{7}$ Sharpe's 1974 paper focuses on the computation of the expected return for a benchmark portfolio. By assuming that the portfolio is ex-ante optimal, Sharpe shows that the vector of expected returns is given by $E\left(\tilde{r}_{B}\right)=\lambda \Omega \tilde{x}_{B}+r_{f}$, where $E\left(\tilde{r}_{B}\right)$ is the vector of expected benchmark returns, $\Omega$ is the covariance matrix, $\tilde{x}_{B}$ is vector of benchmark proportions, $r_{f}$ is the risk-free rate, and $\lambda$ is a normalising constant. ${ }^{8}$

More recent attempts to address the problem of estimating the covariance matrix and the vector of expected returns include among others: Bayesian models for estimation; the MacKinlay and Pastor (2000) missing factor model; the shrinkage estimators of Ledoit and Wolf (2003, 2004a, 2004b); the Kan and Zhou (2007) three-fund model; and the multi-prior model of Garlappi et al. (2007). However, none of these studies focuses on generating portfolios with no short sale positions.

The most intuitive way to overcome the obstacle of short positions is to add short-sale constraints to the portfolio selection problem. Yet, at least from a theoretical point of view, this procedure is problematic, as it generates portfolios with weights that can only be found numerically and not analytically. ${ }^{9}$ Another problem with imposing the short sale constraints, as noted by Black and Litterman (1992), is that they generate 'corner' solutions with zero weights in many assets.

Previous papers by Roll (1977), Roll and Ross (1977), and Rudd (1977) establish analytical conditions on the covariance matrix that give a GMVP with long-only positions. Yet, our main interest in this paper is the tangency portfolio. Kandel (1984) shows that for any set of $N-1$ assets, an $N^{\text {th }}$ asset can be analytically constructed such that the mean variance optimal portfolio will be long-only. However, Levy and Ritov (2001) show that in large markets this $N^{\text {th }}$ asset might be very unrealistic. Green (1986), by employing duality theory, presents conditions that ensure the existence of a long-only mean variance portfolio. These conditions involve the feasibility of portfolios that have non-negative correlation with all assets and positive correlation with at least one. A typical criticism regarding these conditions might be that they are not always constructive enough.

\subsection{This paper}

In this paper we present an additional set of assumptions (to the classical CAPM assumptions) that is sufficient to ensure that the tangency portfolio will indeed 
be long-only. Our additional set of assumptions deals with the structure of the covariance matrix of the risky assets returns and requires the vector of the expected excess returns to be positive. Our new set of assumptions also enables obtaining a long-only GMVP.

The structure we suggest for the covariance matrix is of a two-block type. Namely, we assume that the risky assets can be divided into two blocks, and pairs of risky assets within the same block have the same positive covariance, whereas the covariance between stocks from the two different blocks equals a third negative constant. We then derive analytically sufficient conditions for a matrix of this type to produce a long-only tangency portfolio (as well as a long-only GMVP).

Clearly, in reality the structure of the covariance matrix is not of a two-block type. However, we find this structure to be appealing from the estimation of the covariance matrix prospective, since estimating the two-block covariance matrix requires the estimation of the variances and only three covariances, whereas without imposing any structure on the covariance matrix, its estimation procedure involves the same number of variances, but a huge number of covariances, which can cause a severe estimation error problem. $^{10}$

The idea of reducing the number of parameters that are estimated for constructing the covariance matrix estimator is consistent with many works that have been done in the past. For example, Sharpe (1963) presents the single-index model, which assumes that the co-movement between risky assets is due to a single common influence or index. Elton and Gruber (1973) present the constant correlation coefficient model, which assumes that every pair of risky assets has the same correlation coefficient. Ledoit and Wolf $(2003,2004 a, 2004 b)$ follow the seminal work of Stein (1955) and introduce shrinkage techniques in which the covariance matrix is estimated based on a weighted average of the sample matrix and another covariance matrix estimator on which quite a lot of structure is imposed.

In the next section, we present our theorem which gives an additional set of assumptions that is sufficient to ensure obtaining a long-only tangency/market portfolio (as well as a long-only GMVP).

\section{Theorem}

In this section we show a construct for the covariance matrix that eliminates the problem of tangency portfolios with short sale positions. Our construct - which we call the two-block covariance matrix - assumes that the risky assets can be divided into two groups (blocks). Within each block, the covariance between stocks is identical for all pairs of stocks in the block. The covariance between stocks from different blocks is also identical for all pairs. Thus, in the block structure, the number of covariances associated with each stock is reduced to two: the covariance with the other stocks in the same block (the within-block covariance) and the covariance with the stocks from the other block (the between-block covariance). We will show that this construction leads both to analytical simplicity and incorporates economic logic. We assume a universe of $n$ risky assets and one risk-free asset. 
Definition: A two-block covariance structure has the following form:

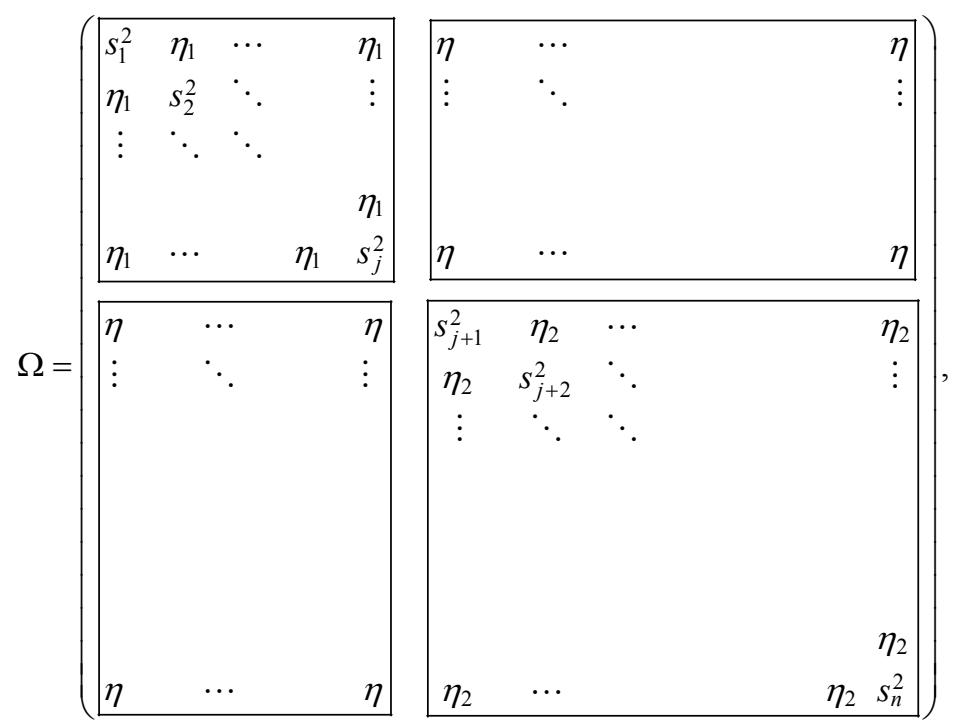

subject to the following conditions:

- The first block is of size $j$ and the second block has size $n-j$.

- $s_{i}^{2}$ are the variances, $\eta_{1}$ and $\eta_{2}$ are the covariances within block 1 and 2 respectively.

- $\quad \eta$ is the covariance between stocks from different blocks.

In the appendix to this paper we prove:

Theorem: Suppose the vector $\mathbf{k}$ of expected excess returns is positive. Then both the tangency portfolio and the GMVP are long-only, if the covariance matrix is two-block and if the following conditions are met:

$$
\begin{aligned}
& 0 \leq \eta_{1}<\min \left(s_{i}^{2}\right) \quad, i=1, \ldots, j \\
& 0 \leq \eta_{2}<\min \left(s_{i}^{2}\right) \quad, i=j+1, \ldots, n \\
& -\sqrt{\left(\eta_{1}+\frac{1}{A}\right)\left(\eta_{2}+\frac{1}{B}\right)}<\eta<\min \left(-\sqrt{\eta_{1}\left(\eta_{2}+\frac{1}{B}\right)},-\sqrt{\eta_{2}\left(\eta_{1}+\frac{1}{A}\right)}\right) \\
& \text { where } A=\sum_{i=1}^{j} \frac{1}{s_{i}^{2}-\eta_{1}} \quad, \quad B=\sum_{i=j+1}^{n} \frac{1}{s_{i}^{2}-\eta_{2}}
\end{aligned}
$$

We can notice that the first two conditions imply that in each block the within-block covariance must be smaller than the variances of all the stocks that are included in this block. Namely, we are dealing here with relatively small-sized within-block covariances. Thus, like the shrinkage estimators advocated by Ledoit and Wolf (2003, 2004a, 2004b), and the portfolios of estimators advocated by Jagannathan and Ma (2000), Disatnik and 
Benninga (2007), and Fletcher (2009), the block matrix has the appealing property of covariance elements which are shrunk compared to the typically large covariances of the traditional sample matrix. Not only are the large covariances those responsible for the extreme short positions that are obtained so often when the mean-variance theory is implemented in practice, but as Michaud (1989) states, inverting the sample matrix with its large covariance elements also amplifies the sampling error.

We can also see that the limitation of the proposed two-block structure is the requirement in the third condition that the between-block covariance has to take a negative value. That is because, in real-life, it can be a challenge to find two groups of stocks that on average move in opposite directions.

\section{Summary}

The classical assumptions of the CAPM do not ensure obtaining a long-only tangency (market portfolio). In this paper, we introduce an additional set of assumptions that is sufficient to ensure that the tangency portfolio will indeed be long-only. Our additional set of assumptions deals with the structure of the covariance matrix of the risky assets returns and requires the vector of the expected excess returns to be positive.

The structure we suggest for the covariance matrix is of a two-block type. We derive analytically sufficient conditions for a matrix of this type to produce a long-only tangency/market portfolio (as well as a long-only GMVP).

We find the structure of a two-block covariance matrix to be appealing from the estimation of the covariance matrix prospective. That is because by imposing this structure, we decrease significantly the number of parameters, which are needed for the estimation process of the covariance matrix. An additional implemental advantage of our two-block construct is that it makes use of relatively small-sized within-block covariances. In future research, we plan to investigate the plausibility of the two-block structure empirically. Specifically, we plan to evaluate the out-of-sample (ex-post) performance of the tangency portfolio that is constructed using a two-block covariance matrix.

\section{References}

Almazan, A., Brown, K., Carlson, M. and Chapman, D. (2004) 'Why constrain your mutual fund manager?', Journal of Financial Economics, Vol. 73, No. 2, pp.289-321.

Benninga, S. (2008) Financial Modeling, 3rd ed., MIT Press, Cambridge, Massachusetts.

Black, F. and Litterman, R. (1990) 'Asset allocation: combining investor views with market equilibrium', Fixed income research, Goldman, Sachs \& Co.

Black, F. and Litterman, R. (1992) 'Global portfolio optimization', Financial Analysis Journal, Vol. 48, No. 5, pp.28-43.

DeMiguel, V., Garlappi, L. and Uppal, R. (2009) 'Optimal versus naïve diversification: how inefficient is the $1 / N$ portfolio strategy?', Review of Financial Studies, Vol. 22, No. 5, pp.1915-1953.

Disatnik, D. and Benninga, S. (2007) 'Shrinking the covariance matrix', Journal of Portfolio Management, Vol. 33, No. 4, pp.55-63.

Disatnik, D. and Katz, S. (2012) 'Portfolio optimization using a block structure for the covariance matrix', Journal of Business Finance and Accounting, Forthcoming. 
Elton, E. and Gruber, M. (1973) 'Estimating the dependence structure of share prices', Journal of Finance, Vol. 28, No. 5, pp.1203-1232.

Fletcher, J. (2009) 'Risk reduction and mean-variance analysis: an empirical investigation', Journal of Business Finance and Accounting, Vol. 36, Nos. 7-8, pp.951-971.

Garlappi, L., Uppal, R. and Wang, T. (2007) 'Portfolio selection with parameter and model uncertainty: a multi-prior approach', Review of Financial Studies, Vol. 20, No. 1, pp.41-81.

Green, R. (1986) 'Positively weighted portfolios on the minimum-variance frontier', Journal of Finance, Vol. 41, No. 5, pp.1051-1068.

Jagannathan, R. and Ma, T. (2000) 'Three methods for improving the precision in covariance matrix estimation', Unpublished working paper.

Jagannathan, R. and Ma, T. (2003) 'Risk reduction in large portfolios: why imposing the wrong constraints helps', Journal of Finance, Vol. 58, No. 4, pp.1651-1684.

Kan, R. and Zhou, G. (2007) 'Optimal portfolio choice with parameter uncertainty', Journal of Financial and Quantitative Analysis, Vol. 42, No. 3, pp.621-656.

Kandel, S. (1984) 'On the exclusion of assets from tests of the mean variance efficiency of the market portfolio', Journal of Finance, Vol. 39, No. 1, pp.63-75.

Ledoit, O. and Wolf, M. (2003) 'Improved estimation of the covariance matrix of stock returns with an application to portfolio selection', Journal of Empirical Finance, Vol. 10, No. 5, pp.603-621.

Ledoit, O. and Wolf, M. (2004a) 'A well-conditioned estimator for large-dimensional covariance matrices', Journal of Multivariate Analysis, Vol. 88, No. 2, pp.365-411.

Ledoit, O. and Wolf, M. (2004b) 'Honey, I shrunk the sample covariance matrix', Journal of Portfolio Management, Vol. 30, No. 4, pp.110-119.

Levy, M. and Ritov, Y. (2001) 'Portfolio optimization with many assets: the importance of short-selling', Working paper, the Hebrew University of Jerusalem.

Lintner, J. (1965) 'Security prices, risk, and the maximal gains from diversification', Journal of Finance, Vol. 20, No. 4, pp.587-615.

MacKinlay, A. and Pastor, L. (2000) 'Asset pricing models: implications for expected returns and portfolio selection', Review of Financial Studies, Vol. 13, No. 4, pp.883-916.

Markowitz, H. (1952) 'Portfolio selection', Journal of Finance, Vol. 7, No. 1, pp.77-91.

Markowitz, H. (1959) Portfolio Selection: Efficient Diversification of Investments, Yale University Press, New Haven, CT.

Michaud, R. (1989) 'The Markowitz optimization enigma: is 'optimized' optimal?', Financial Analyst Journal, Vol. 45, No. 1, pp.31-42.

Mossin, J. (1966) 'Equilibrium in a capital asset market', Econometrica, Vol. 34, No. 4, pp.768-783.

Roll, R. (1977) 'A critique of the asset pricing theory's tests; Part I: on past and potential testability of the theory', Journal of Financial Economics, Vol. 4, No. 2, pp.129-176.

Roll, R. and Ross, S. (1977) 'Comments on qualitative results for investment proportions', Journal of Financial Economics, Vol. 5, No. 2, pp.265-268.

Rudd, A. (1977) 'A note on qualitative results for investment proportions', Journal of Financial Economics, Vol. 5, No. 2, pp.259-263.

Sharpe, W. (1963) 'A simplified model for portfolio analysis', Management Science, Vol. 9, No. 2, pp.277-293.

Sharpe, W. (1964) 'Capital asset prices: a theory of market equilibrium under conditions of risk', Journal of Finance, Vol. 19, No. 3, pp.425-442.

Sharpe, W. (1974) 'Imputing expected security returns from portfolio composition', Journal of Financial and Quantitative Analysis, Vol. 9, No. 3, pp.463-472. 
Stein, C. (1955) 'Inadmissibility of the usual estimator for the mean of a multivariate normal distribution', Proceedings of the 3rd Berkley Symposium on Probability and Statistics, University of California Press, Berkeley, pp.197-206.

\section{Notes}

1 See, for example, Ledoit and Wolf (2003), Jagannathan and Ma (2003), Disatnik and Benninga (2007), and DeMiguel et al. (2009).

2 See, for example, regulation SHO of the US Securities and Exchange Commission (SEC), http://www.sec.gov/spotlight/shortsales.htm. Almazan et al. (2004) report that over the $1994-2000$ period $69 \%$ of their sample of US domestic equity mutual funds were not allowed to short.

3 Note that in practice the GMVP often includes less extreme short sale positions than other efficient portfolios [see for example, Jagannathan and Ma (2003)]. Nevertheless, these short sale positions are still quite significant [see for example, Disatnik and Benninga (2007) and Disatnik and Katz (2012)].

4 The reasonable correspondence of implied option volatilities with the asset return variances leads one to believe that historical data may be a better predictor of variances.

5 With the exception of Kellogg $(\mathrm{K})$ all of the historical average returns are implausibly high as a prediction of future expected returns. This is another problem of using historical data.

6 In examining these short-cut methods to compute the covariance matrix, it is sometimes difficult, from the current vantage point, to distinguish between the need for greater computational efficiency and the realisation that the historical data gives problematic outcomes in the optimisation process.

7 This method became the foundation of the Black-Litterman (1990) model.

$8 \lambda$ is often taken to be a constant representing the optimiser's risk aversion. Benninga (2008) shows that $\lambda$ can be interpreted in relation to the expected benchmark return.

9 To obtain a solution for the constrained optimisation problem, an iterative procedure, based on the Kuhn-Tucker conditions, is commonly used.

10 For example, when portfolios of 150 risky assets are considered, without imposing a structure on the covariance matrix, one has to estimate 11,175 different covariances.

\section{Appendix}

\section{The Proof of the Theorem}

The proof consists of three parts. First, we show that, under the conditions of the theorem, the two-block structure indeed generates an invertible covariance matrix. Then, we show that, under these conditions, a long-only GMVP is obtained. Lastly, we show that, under the same conditions, also a long-only tangency portfolio is obtained.

- Part 1: Disatnik and Katz (2012) show that the two-block matrix is positive definite for: 


$$
\begin{aligned}
& 0 \leq \eta_{1} \leq \min \left(s_{i}^{2}\right), \quad i=1, \ldots, j \\
& 0 \leq \eta_{2} \leq \min \left(s_{i}^{2}\right), \quad i=j+1, \ldots, n \\
& -\sqrt{\left(\eta_{1}+\frac{1}{A}\right)\left(\eta_{2}+\frac{1}{B}\right)}<\eta<+\sqrt{\left(\eta_{1}+\frac{1}{A}\right)\left(\eta_{2}+\frac{1}{B}\right)}
\end{aligned}
$$

Therefore, under the conditions of the theorem, we always obtain a positive definite matrix. This implies that the two-block matrix is indeed an invertible covariance matrix, since it is also a symmetric matrix.

- Part 2: Given a covariance matrix $\boldsymbol{\Omega}$, the vector of the GMVP weights is obtained by $\frac{\Omega^{-1} 1}{\mathbf{1}^{\mathrm{T}} \boldsymbol{\Omega}^{-1} \mathbf{1}}$, where $\boldsymbol{\Omega}^{-\mathbf{1}}$ denotes the inverse matrix of $\boldsymbol{\Omega}, \mathbf{1}$ denotes a vector of ones, and $\mathbf{1}^{\mathrm{T}}$ denotes the transpose vector of $\mathbf{1}$. Using the two-block structure of $\boldsymbol{\Omega}$, Disatnik and Katz (2012) show that the weight of stock $i$ in the GMVP that $\mathbf{\Omega}$ produces is:

$$
\begin{aligned}
& w_{i}=\frac{1}{s_{i}^{2}-\eta_{1}} \cdot \frac{1+\left(\eta_{2}-\eta\right) B}{A+B+\left(\eta_{1}+\eta_{2}-2 \eta\right) A B}, i=1, \ldots, j \\
& w_{i}=\frac{1}{s_{i}^{2}-\eta_{2}} \cdot \frac{1+\left(\eta_{1}-\eta\right) A}{A+B+\left(\eta_{1}+\eta_{2}-2 \eta\right) A B}, i=j+1, \ldots, n
\end{aligned}
$$

Thus, we can see that a long-only GMVP is generated, if the following set of conditions holds:

$$
\begin{aligned}
& \eta_{1}<\min \left(s_{i}^{2}\right), i=1, \ldots, j \\
& \eta_{2}<\min \left(s_{i}^{2}\right), i=j+1, \ldots, n \\
& \eta \leq \min \left(\eta_{1}, \eta_{2}\right)
\end{aligned}
$$

Therefore, under the conditions of the theorem, we always obtain a long-only GMVP.

- Part 3: Since by assumption the vector of excess returns, $\mathbf{k}$, is positive, in order to find a long-only tangency portfolio, whose vector of weights is given by $\frac{\mathbf{\Omega}^{-1} \mathbf{k}}{\mathbf{1}^{\mathbf{T}} \mathbf{\Omega}^{-1} \mathbf{k}}$, it is sufficient to prove that under the conditions of our theorem all the elements of $\mathbf{\Omega}^{\mathbf{- 1}}$ are positive.

Symmetric considerations imply that, given the two-block structure of $\mathbf{\Omega}$, under the conditions of the theorem, in $\mathbf{\Omega}^{-\mathbf{1}}$ : all the diagonal elements have the same sign; all the off-diagonal elements in the first block have the same sign; all the off-diagonal elements in the second block have the same sign; and all the elements outside the two blocks have the same sign.

We progress by showing that when $\eta<0$, all the elements outside the two blocks of $\boldsymbol{\Omega}^{\mathbf{- 1}}$ are positive. By explicitly writing the equations for $\boldsymbol{\Omega} \boldsymbol{\Omega}^{\mathbf{- 1}}=\mathbf{I}$, where $\mathbf{I}$ denotes the identity matrix, it is straightforward to show that when $\eta=0$, all the elements 
outside the two blocks equal zero. Saying that the GMVP is long-only is equivalent to saying that the sum of the elements in each of the columns of $\boldsymbol{\Omega}^{-\mathbf{1}}$ is positive. As a result, it can be shown that the elements outside the two blocks cannot equal zero, unless $\eta=0$. Using again that $\boldsymbol{\Omega}^{\mathbf{- 1}}=\mathbf{I}$ enables to show that when all the elements in $\mathbf{\Omega}^{\mathbf{- 1}}$ are positive, $\eta$ must be negative. Adding the property that all the elements in $\mathbf{\Omega}^{\mathbf{- 1}}$ are continuous functions of $\eta$ enables to show that when $\eta \rightarrow 0^{+}$, all the elements outside the two blocks are negative. Combining the last three results with the abovementioned continuous property implies that when $\eta<0$, all the elements outside the two blocks of $\boldsymbol{\Omega}^{-1}$ must be positive.

We now turn to the off-diagonal elements in the two blocks of $\mathbf{\Omega}^{\mathbf{- 1}}$. It can be shown that, under the conditions of the theorem, when $\eta=-\sqrt{\eta_{1}\left(\eta_{2}+\frac{1}{B}\right)}$, all the offdiagonal elements in the first block equal zero and when $\eta=-\sqrt{\eta_{2}\left(\eta_{1}+\frac{1}{A}\right)}$, all the off-diagonal elements in the second block equal zero.

It can also be shown that in the range $\left(-\sqrt{\left(\eta_{1}+\frac{1}{A}\right)\left(\eta_{2}+\frac{1}{B}\right)},-\sqrt{\eta_{1}\left(\eta_{2}+\frac{1}{B}\right)}\right)$, the off-diagonal elements in the first block are decreasing functions of $\eta$, and in the range $\left(-\sqrt{\left(\eta_{1}+\frac{1}{A}\right)\left(\eta_{2}+\frac{1}{B}\right)},-\sqrt{\eta_{2}\left(\eta_{1}+\frac{1}{A}\right)}\right)$, the off-diagonal elements in the second block are decreasing functions of $\eta$. As a result under the conditions of the theorem, all the off-diagonal elements in both blocks of $\boldsymbol{\Omega}^{-\mathbf{1}}$ are positive.

Lastly, after obtaining that under the conditions of the theorem all the off-diagonal elements in $\mathbf{\Omega}^{-\mathbf{1}}$ are positive, it is straightforward to show, using again the equations derived from $\mathbf{\Omega} \boldsymbol{\Omega}^{-\mathbf{1}}=\mathbf{I}$, that also the diagonal elements in $\boldsymbol{\Omega}^{-\mathbf{1}}$ are positive. 